\title{
Evidence-Based Valuation in Oncology: Lessons Learned from a Case Study
}

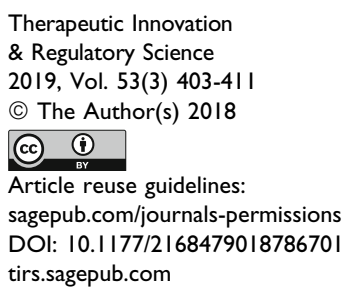

2019, Vol. 53(3) 403-4II

(C) The Author(s) 2018

(c) (i)

sagepub.com/journals-permissions

tirs.sagepub.com

\author{
John J. Doyle, Dr PH, MPH' $\odot$, Emily Hawryluk, BS ${ }^{2}$, \\ Jeffrey Niemira, BA $^{3}$, and Brian Wood, MBA ${ }^{3}$
}

\begin{abstract}
Background: Value demonstration in health care remains a challenge. This paper examines traditional approaches to pricing and the evolution of value-based pricing (VBP), and proposes a new framework for evidence-based valuation (EBV). The main objective of $E B V$ is to estimate the value-based pricing range for the new medicine, identify key product attributes that drive value as perceived by various stakeholders, and then elucidate the requisite evidence to support those value claims.

Methods: EBV centers on a structured framework for estimating a drug's price based on its perceived value to various stakeholders. The EBV framework consists of identifying key value attributes that drive adoption of a drug in a given therapeutic area; gaining insights into stakeholder value considerations and evidence requirements; and quantifying stakeholders' perceptions of specific value attributes within pricing premiums.

Results: An example demonstrates the application of the EBV framework in a simplified manner for 3 drugs indicated for renal cell carcinoma, 3 drugs for prostate cancer, and I drug for melanoma. HTAs, published trial results, and publications archived in PubMed between 2005 and 2013 were analyzed to identify key value attributes. The following 5 attributes were considered: overall survival (OS), progression-free survival (PFS), population size, trial comparator, and adverse events.

Conclusions: The method described offers a means to appraise pharmaceuticals in an environment increasingly focused on evidence-based medicine and value-based health care.
\end{abstract}

\section{Keywords}

evidence-based value framework, value demonstration in health care, pricing in oncology

\section{Background}

Value in health care is a conundrum in many countries. Unlike the situation with most non-health care products and services, market forces between the end-consumer and the seller do not allow the market to work to determine optimal prices for health care services. In developed countries, patients often have no idea how much their treatments cost, and therefore have no incentive to seek value. This responsibility therefore often falls on a third party payer.

Globally, payers are slowly moving toward paying for health outcomes rather than utilization of products and services. There is a spectrum of activity-ranging from the centralized process of Health Technology Assessments (HTAs) to the decentralized use of risk-based contracts - all geared to measure and compensate based on real-world performance.

The ongoing transformation in the United States from volume-based to value-based care is illustrated by developments such as the Hospital Value-based Purchasing Program, readmission penalties, bundled payments to physicians, and Accountable Care Organization shared savings programs. Complementing this is payers' increased use of value-based purchasing of provider services and value-based insurance design for new benefit packages. The intent of these initiatives is to channel patients to higher value care providers, services, and products.

Similarly, there is an increasing push from regulators to apply structured quantitative methodologies for benefit-risk assessments, with multiple regulatory and industry-wide initiatives underway. These share common elements in defining the decision problem and therapeutic context, identifying key benefit and risk factors, and interpreting and communicating the benefit-risk findings. The authors have investigated published quantitative benefit-risk frameworks, finding a lack of standardization and opportunity for developing a harmonized framework that could be applied across different therapeutic areas.

\footnotetext{
' IQVIA, New York, NY, USA

${ }^{2}$ IQVIA, Durham, NC, USA

${ }^{3}$ IQVIA, Boston, MA, USA
}

Submitted 19-Dec-2017; accepted 22-May-2018

Corresponding Author:

John Doyle, Dr PH, MPH, IQVIA, One Grand Central Place, 60 East 42nd Street, Suite 2230, New York, NY 10165, USA.

Email: john.doyle@iqvia.com 
Value demonstration in health care is a continued challenge. Even when earnest attempts are made to focus on and evaluate just a few of the variables in play, the myriad interconnected components in the system complicate the picture quickly. As with evidence-based medicine, providers and payers are beginning to interact in a new way that utilizes real-world, empirical data and advanced analytics more often. Payers are on a path to provide reimbursement based on quality metrics and outcomes. In this way, real-world data are helping to align incentives between providers and payers around patients, and permitting more frequent implementation of value-based decisions.

In oncology in particular, the extremely high prices of innovative therapies frequently hit the headlines, with recently approved CAR-T cell therapies close to $\$ 500,000 .{ }^{1}$ Patients and physicians have responded to such pricing with anger and dismay. A recent poll found that 6 of 10 Americans ranked drug prices as their top priority for government action. ${ }^{2}$

\section{Shift in Influence}

Participants in the industry must respond to growing health care costs and intense scrutiny of pricing. This is a reflection of increased per-unit price as well as increased volume based on escalating diagnosed incidence and prevalence of many diseases - against a backdrop of fruitful innovation. Most notably, there appears to be a shift in the way oncology value is perceived, from a position where the price and value of therapies were rarely questioned, to a broader debate around the health economic and societal value of each therapy. Manufacturers increasingly have to demonstrate to stakeholders the value of their product in particular disease settings and market niches. It is no longer simply about price to the payer; specifically, in the U.S., this is evident through the substantial changes in benefit design by many private payers over the past decade. For instance, the increased use of specialty pharmaceutical pricing tiers results in increased economic burden and out-of-pocket expenses being borne by patients, such that there is justifiable concern regarding affordability and whether society is truly accessing new technology in an equitable way.

\section{Background and Evolution of Value-Based Pricing}

Value is the utility gained from the use of a product. A valuebased price is one that accurately reflects the utility gained by the end-user of the product, and in many cases the societal utility when considered from a broader population health perspective. Value can be derived by understanding the relative cost of a product vis-à-vis competition, the opportunity cost of foregone alternative options, and the economic impact of the total cost to the health care system.

"Everything is worth what its purchaser will pay for it" - Publilius Syrus

Incremental utility can be gained through both physiological and psychological effects, since both create incremental welfare for the user. For pharmaceuticals, net benefits have traditionally been captured through health economic analysis. This aims to estimate cost differentials or health gain accrued by use of a product over time versus a comparator.

The health care community is still undecided on how to convert health benefits to monetary value, so the standard output is typically left as a cost per unit of health improvement or net health benefit compared to willingness to pay for specific types of health improvements. ${ }^{3}$ While physiologic effects are often captured with laboratory testing and clinical assessment, psychological effects have historically been collected via structured surveys of either patients or health care providers. Unlike blood pressure, hemoglobin levels, or body mass index, psychological benefit requires more qualitative research methods to arrive at a quantifiable value and is highly patient specific. ${ }^{4}$ The translation of value, although ultimately realized by the patient, must be demonstrated to a third party, often the government or insurer, in order to achieve an agreeable price. ${ }^{5}$

Value-based pricing (VBP) for medicines is not a new concept, and value-based pricing techniques have been employed in countries that utilize formal HTAs, such as Australia, Canada, the UK, and the Netherlands. Both Germany (under AMNOG) ${ }^{6}$ and France ${ }^{7}$ demand "added-value" to be demonstrated through comparative outcomes (directly or indirectly) in order for a drug to obtain premium pricing. ${ }^{8}$ Similarly, emerging markets are beginning to integrate the processes and ideas of HTAs into their decision-making to justify value-based pricing and reimbursement. ${ }^{9}$

In the past three years several alternative drug value frameworks have emerged, highlighting a renewed interest in this area. In the U.S., these include the American Society of Clinical Oncology's (ASCO's) Value Framework, the National Comprehensive Cancer Network's (NCCN's) Evidence Blocks, Memorial Sloan Kettering Cancer Center's (MSKCC's) DrugAbacus, and the Institute for Clinical and Economic Review's (ICER's) Value Assessment Framework. Each of these was introduced to allow stakeholders to appraise therapies based on their value in a transparent and objective manner. Although each has applicability, there are limitations that restrict their individual applicability as a pricing benchmark, and as we have shown in past work, there are often inconsistencies in the results they produce. ${ }^{10}$

\section{Traditional Approaches to Pricing}

Pharmaceutical manufacturers have historically approached pricing by balancing the needs of internal stakeholders who are concerned with return on investment in research and development of innovative medicines, and external stakeholders who want to see clinical and economic differentiation to justify the price. Traditional pricing methods include reference price benchmarking, willingness-to-pay assessment and cost effectiveness analysis, each of which approaches this balance in a slightly different way.

As depicted in Figure 1, each traditional pricing method has specific strengths, and weaknesses that can contribute to 


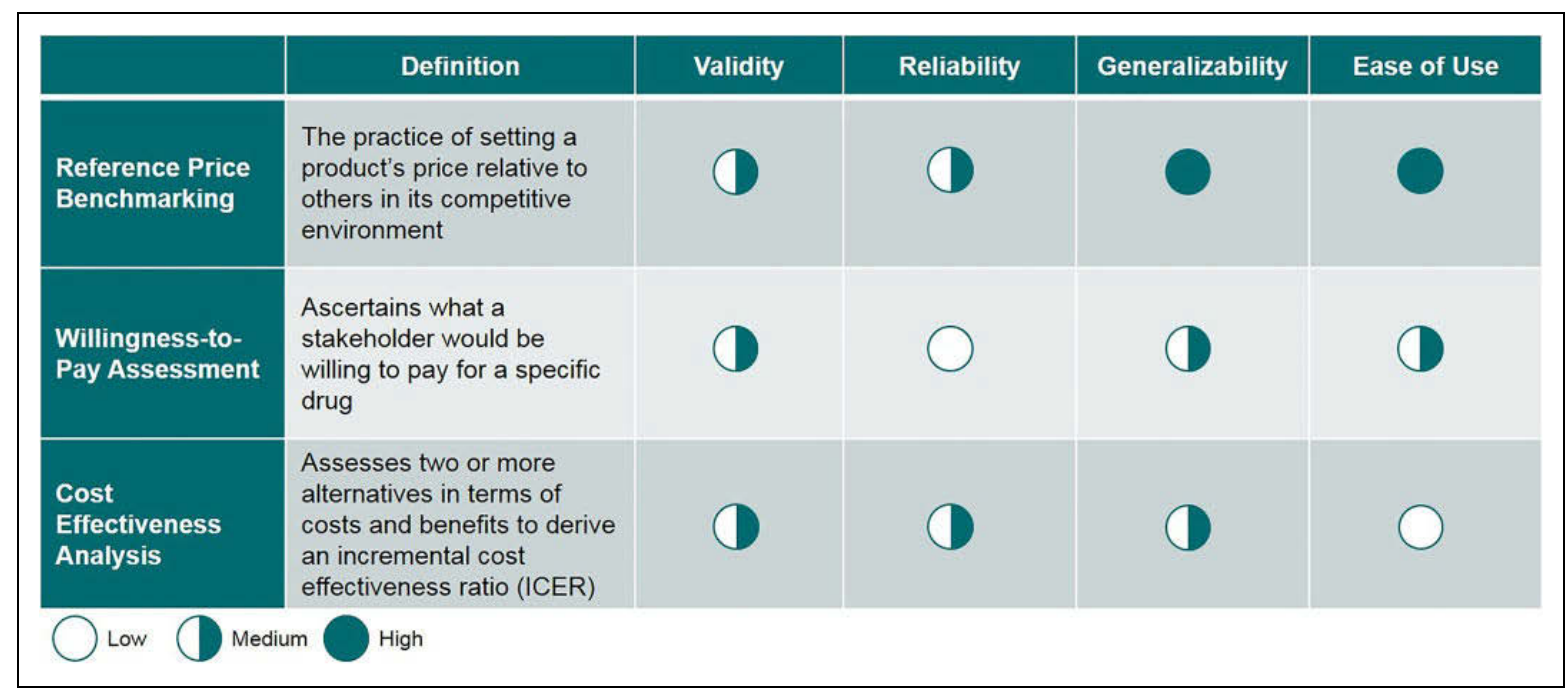

Figure I. Comparison between various approaches to pricing.

imprecise and inaccurate pricing. One way to minimize the risk of a potentially inaccurate price is to adopt complementary aspects of all 3 pricing methodologies, and merge them into one robust, reliable, and generalizable pricing model: the evidence-based valuation (EBV) model. The following sections will further explore this EBV methodology and its unique approach to triangulating across multiple methods to pricing.

\section{Methods}

\section{An Approach to Value Pricing}

An evidence-based approach to valuation in pharmaceuticals (EBV) centers on a structured framework for estimating the price of a drug based on its perceived value by various stakeholders, fortified by proof points that quantify the differentiation of the new agent from a selected comparator. The main objective of EBV is not only to estimate the value-based price range for the new medicine, but also to identify key product attributes that drive value as perceived by various stakeholders and then elucidate the evidence required to support those value claims. The EBV framework consists of

1. Identifying key value attributes that drive adoption of a drug in a given therapeutic area

2. Gaining insights into stakeholder value considerations and evidence requirements

3. Quantifying stakeholders' perceptions of specific value attributes within pricing premiums.

Each of these elements is considered in turn below.

I. Identifying key value attributes that drive drug adoption in a therapeutic area

A robust method begins with a thorough review of historical HTA decisions, published literature, and market research pertaining to health care system stakeholders. Health care agencies worldwide routinely use centralized assessments to make informed decisions concerning reimbursement of medicines and other health technologies. Evaluating decisions by various HTA bodies provides assessment information such as outcome measures, clinical and economic outcomes, final recommendations, and insights into their relative importance.

Based on findings from HTA analyses and secondary research, attributes are identified that are important to decision-making bodies and drive the value of new assets. The selected attributes are then segmented into various categories such as clinical, economic, and humanistic elements. These vary by disease indication and type of product being evaluated, but examples include metrics such as overall survival and progression-free survival in cancer care.

\section{Gaining insights into stakeholder value considerations and} evidence requirements

The definition of value differs between various stakeholder groups, and an understanding of each group's perception of value is required to develop an evidence-based pricing strategy. These stakeholders include patients, physicians, payers, policymakers, and pharmaceutical companies - who are all seeking treatments that offer the best risk-benefit and cost-benefit ratios. Stakeholders weigh risks and benefits differently, and incur varied costs. Perceptions of value can also differ between individuals within these stakeholder groups.

For patients, quality of life, symptomatic improvement, lack of side effects, and convenience of dosage may be most important. Out-of-pocket costs under health insurance plans may also be a factor. Physicians typically seek evidencebased, effective diagnostic and therapeutic interventions that are delivered efficiently. For payers and policy makers, the key trade-offs involve allocation of resources, taking into account a therapy's ability to improve health outcomes, quality of life and productivity, and to reduce the total cost of care. There is often a focus on quantifiable measures such as price 
and real-world outcomes. Pharmaceutical companies wish to see continued incentives for development of innovative products with a positive impact on population health in terms of costs and benefits over time.

The FDA is placing an increasing focus on patient preferences. The Center for Devices and Radiological Health (CDRH) has set up a Patient Preference Initiative (PPI) to advance the science of measuring these preferences, so that the patient voice can be incorporated more broadly into decision making. In recent examples, the Center for Drug Evaluation and Research (CDER) encouraged a patient advocacy group to propose draft guidance on engaging stakeholders in regulatory decision making for muscular dystrophy, and CDRH sponsored a discrete-choice experiment case study to quantify obese respondents' perspectives on meaningful benefits. ${ }^{11}$ The European Medicines Agency is also seeking to collect individual patient preferences to inform regulatory review, including a pilot study to obtain patient input. ${ }^{12}$ The EU Committee for Medicinal Products for Human Use (CHMP) also has in place procedures to include patient perspectives within the EMA's assessments of benefit-risk. ${ }^{13}$ In addition, a recent guideline on benefit-risk information in drug development and regulation from the International Council for Harmonisation (ICH) "opens the possibility to include information about patient perspectives, for example information from patient preference studies."

Understanding stakeholder value considerations can be achieved by prioritizing a set of attributes for each stakeholder, capturing their perception of value, and understanding the importance or relevance of each attribute to their decision making. The efficacy and safety profile of the product for which the EBV recommendation is being developed is validated with stakeholders through surveys or qualitative interviews to understand unmet needs and to obtain preliminary reactions to the proposed price.

\section{Quantifying stakeholders' value perceptions of pricing premiums}

A rigorous quantitative study or choice exercise should be conducted to determine the relative importance of attributes. In this exercise, stakeholders are presented with multiple hypothetical profiles created by various attribute values to obtain their willingness to pay for each profile. Stakeholders' feedback and responses gained during this research are then analyzed through methodologies such as conjoint analysis and Bayesian modeling to derive the contribution of individual attributes to overall willingness to pay. These methods are utilized to convert actual survey responses (stated) into price premiums (derived). The various quantified values are then used as inputs to a VBP model, which translates them into a preliminary pricing range.

A variety of approaches may be used to obtain the stakeholders' valuation of product attributes. The choice depends on the complexity of the market, the ability to obtain sufficient sample size for survey research, and the scope of the research. Methods involve estimating the log-linear model (in rare cases, such as when the agent being evaluated differs significantly from the available comparators, a linear price model may be more appropriate):

$$
\log (P)=B X+C+\varepsilon
$$

where $P$ is the price, $B$ is a vector of utilities associated with the attributes, $X$ is a vector of product attributes, $C$ is a constant term, and $\varepsilon$ is the error term. This equation relates each product attribute to a percentage change in price and works well for reasonable price ranges. It is also very useful for doing analysis across markets/indications where the prices may differ.

The simplest approaches include explicit pricing techniques. For example, payer respondents state what they are willing to pay for a product with a set of attributes or assign a price premium to the difference between products or attributes. The approach is easy to understand for both respondents and researchers and the modeling is straightforward. The model is fitted directly to the survey responses, typically requiring a smaller sample size than alternative approaches. This method is hindered by concern that respondents may overstate their price sensitivity (eg, understate the price premiums). Explicit pricing techniques are suboptimal, but are an option when sample sizes are constrained.

Derived pricing methods are generally preferred to explicit methods. Respondents evaluate a series of products at varying hypothetical prices. The valuations mimic the market decisions made by the stakeholder. Physicians may be asked for what proportion of eligible patients they would prescribe such a product; payers may be asked at which level they would anticipate reimbursing such a product. The approach reduces bias by forcing respondents to make trade-off decisions. Emulating actual market decisions can have undesired consequences. Price sensitivities may reflect market dynamics more than a product's perceived value. For example, the impact of price on a physician's decision making may not reflect the perceived value of the product if reimbursement practices are very burdensome or very permissive. Trade-off exercises that are more detached from actual market decisions, such as max-diff, can mitigate this limitation.

Modeling data from derived pricing exercises requires an additional step compared to the explicit pricing exercises. Stakeholder decisions are assumed to be a function of the product's utility:

$$
U(X, P)=B X+A \cdot \log (P)+C+\varepsilon
$$

Holding utility constant, the desired equation

$$
\log (P)=B X+C
$$

can be retrieved. More commonly, the utility function is used to compare the product to an existing product

$$
\log (P)-\log \left(P_{c}\right)=B X-B X_{c}
$$

where $P_{c}$ is the price of the comparator and $X_{c}$ is a vector of the comparator attributes. This equation calculates the price premium at which respondents show no preference between the product and the comparator. We describe this as the differentiation premium. 


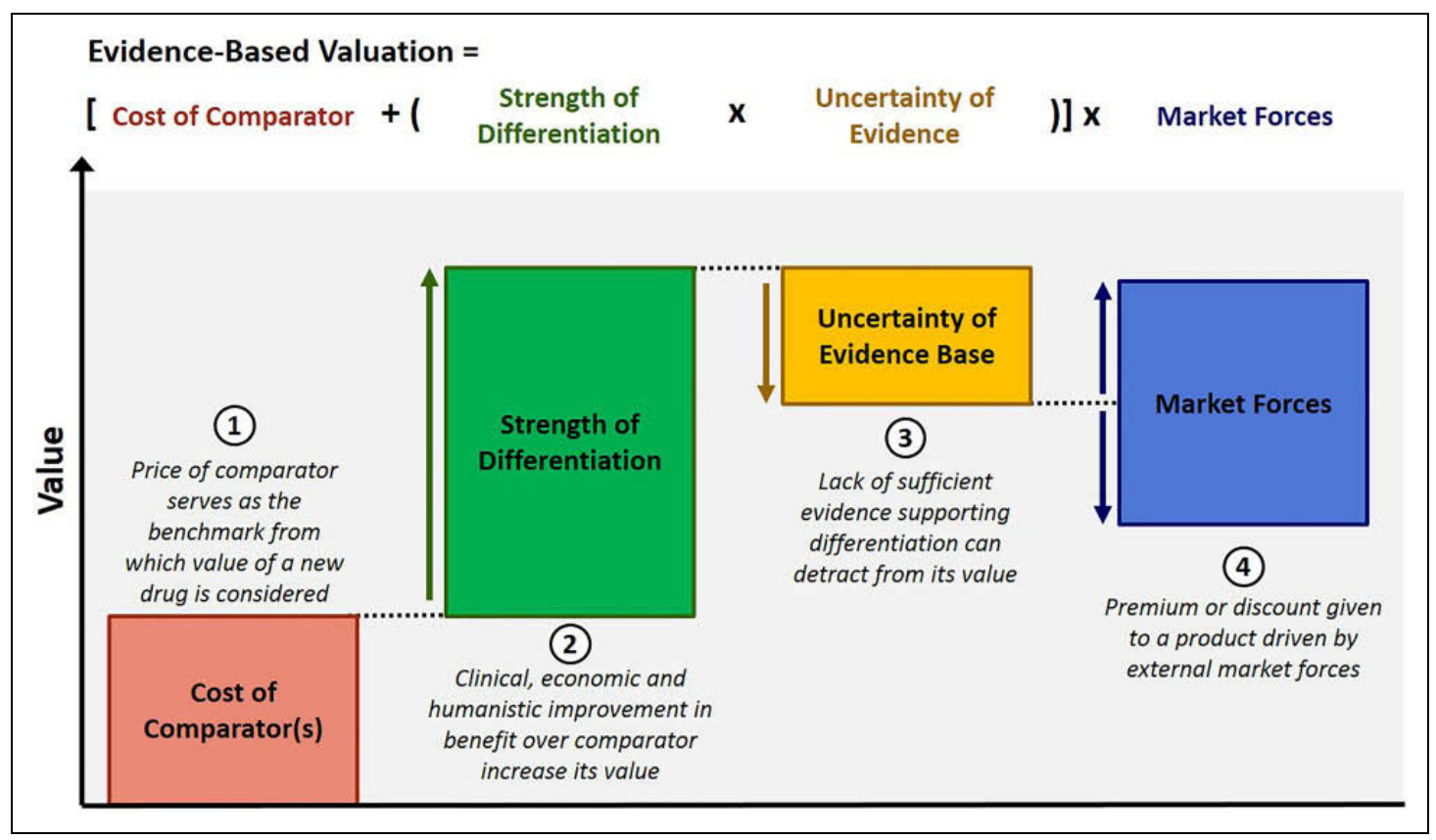

Figure 2. An evidence-based valuation.

\section{An Evidence-based Valuation Equation}

The economic data collected from the VBP process discussed above is then populated into a pricing equation to calculate a product's preliminary value-based pricing range (Figure 2).

The EBV equation comprises 4 variables that affect a product's pricing potential. The foundational variable is "competitor price," which forms the basis or reference point, and each additional variable contributes to an increase or decrease in the product's value based on the asset profile and market conditions, resulting in a value-based pricing range for the target asset.

\section{Equation Variables Explained in Detail}

Competitor Price: Cost of selected competitor, dependent on its perceived placement in the treatment algorithm

Since the price of competitor products forms the basis of the VBP equation, it is critical to select the relevant competitor for comparison. This does not necessarily have to be a product used to treat the same condition, although this is preferable unless no drug currently exists to treat the condition, in which case a drug from another similar disease or indication must be chosen. Ideally, the standard of care is selected for comparison purposes, but payers are also known to anchor value assessments to market analogs where there is no obvious choice for reference product. Our EBV framework allows the user to choose from a set of relevant competitors and compare the incremental value offered by the target asset against any of those competitors.

Differentiation Adjustment: Clinical and economic differentiation from selected competitor and associated pricing premium The selected attributes are separated into clinical, economic, and humanistic categories. Based on stakeholders' self- expressed utilities and quantified willingness to pay for 1 unit of incremental value of an attribute, willingness to pay in terms of percentage premium can be estimated by comparing the absolute attribute values of selected competitor and the target asset. Each attribute's contribution adds up to the differentiation variable's contribution to the equation.

Evidence Quality Uncertainty Adjustment: "Believability" of trial evidence as determined by the strength of methods used to collect the evidence

The trial evidence presented to stakeholders may not be the gold standard or meet stakeholders' expectations. There could be differences in opinion about the trial comparator, various elements of the trial design, or health economic assumptions, and stakeholders may devalue differentiation evidence as a result. Based on the valuation provided by stakeholders for various trial design scenarios, the quality of the target asset's trial design evidence can be estimated and applied to the differentiation variable to devalue the evidence-based value price.

Market Adjustment: Premium or discount given to a product's intangible value that is driven by the market rather than the manufacturer

This variable allows for an adjustment based on marketspecific factors. It also allows for recalibration of pricing potential as market forces change as a result of various economic or social events in the future. The market adjustment is governed by many factors such as the ability of the health system to pay for expensive drugs, unmet need in the market, and competitive intensity. For an asset, even though differentiation could be same across markets because of a similar 
Therapeutic Innovation \& Regulatory Science 53(3)

product profile, the willingness and ability to pay for the same drug might be different as a result of varying market forces.

\section{Results}

\section{Applying the EBV Framework: A Case Study}

The example below demonstrates the application of this EBV framework in a simplified manner. In our analysis, we included 3 drugs indicated for renal cell carcinoma (RCC; drugs A, B, C), 3 drugs indicated for prostate cancer (drugs D, E, and F), and 1 drug indicated for melanoma (drug G). HTAs, published trial results, and publications archived in PubMed between 2005 and 2013 were analyzed to identify key value attributes. The following 5 attributes were considered:

- Overall survival (OS)

- Progression-free survival (PFS)

- Population size

- Trial comparator

- Adverse events

Utilizing outcomes data from clinical trials for each of the 5 drug attributes, the performance of each of the 7 oncologic therapies was measured with respect to the selected attributes.

An aggregate value score was generated for each drug using the selected attributes based on the published trial results and after assigning scores based on qualitative criteria (refer to Table 1 for scoring criteria and Table 2 for value scores). The cost per month of each drug in the US was calculated based on dosing and published wholesale acquisition cost (WAC). Drugs were then compared on value score vs cost per month to understand the value-cost relationship.

A logarithmic curve was fitted to the data to derive payer evidence thresholds based on the value offered by selected products at various prices. Each drug's expected value-based price range was estimated based on its value score along the log curve. This curve can be plotted for any market or therapeutic area. Figure 3 shows the value vs cost relationship of the 7 oncologic therapies.

\section{Discussion}

\section{Relevance of the EBV Framework}

Value in healthcare remains largely unmeasured and misunderstood...the creation of value for patients should determine the rewards for all other actors in the system. - Michael Porter

As payers struggle to balance their health care spending and manufacturers face decisions regarding investment in new drug development, the need for a more transparent and evidencebased method to determine value in health care is crucial. Facing a level of complexity that is unique to health care, the market contains third parties such as governments and private insurers, who participate to varying degrees depending on the relevant health care system. One traditional method proposed
Table I. Product Attribute Weighting and Scoring Criteria.

\begin{tabular}{lcccc}
\hline Attribute & Woighting* & $\begin{array}{c}\text { Low } \\
100\end{array}$ & $\begin{array}{c}\text { Medium } \\
200\end{array}$ & $\begin{array}{c}\text { High } \\
300\end{array}$ \\
\hline $\begin{array}{l}\text { Overall survival (OS) } \\
\text { Progression-free survival }\end{array}$ & $35 \%$ & $\leq 1.2 x$ & $>1.2 x-1.5 x$ & $>1.5 x$ \\
$\quad$ (PFS) & $15 \%$ & $\leq 1.2 x$ & $>1.2 x-1.5 x$ & $>1.5 x$ \\
Population size & $20 \%$ & $<5 \%$ & $5 \%-20 \%$ & $>20 \%$ \\
$\begin{array}{l}\text { Trial comparator } \\
\text { Adverse events (grade 3/4) }\end{array}$ & $15 \%$ & Placebo & & SOC \\
Total & $15 \%$ & Low & Medium & High \\
& $100 \%$ & & & \\
\hline
\end{tabular}

Table 2. Value Score Calculations.

\begin{tabular}{lcc}
\hline Drug & Cost per Month & Value Score \\
\hline A & $\$ 9000$ & 180 \\
B & $\$ 7000$ & 130 \\
C & $\$ 8900$ & 185 \\
D & $\$ 10600$ & 220 \\
E & $\$ 7750$ & 200 \\
F & $\$ 5000$ & 175 \\
G & $\$ 10000$ & 270 \\
\hline
\end{tabular}

for determining value is to use cost-effectiveness analysis or to set budgetary thresholds for how much payers are willing to pay. Although third party payers may not be the end users of the drug, typically, their objective is to provide health care to constituents at a price that is consistent with product value. Only by adequately quantifying the benefits and costs of a therapy can a value-based price be determined.

In the US, this can be observed in the relatively novel concept of value-based insurance design (VBID). The goal of VBID is to encourage the use of health care services that provide greatest value to patients while not reducing the patient's choice set. Definitions of value are again subject to interpretation, further highlighting the need for a robust and transparent methodology such as that described above. Unlike a policy that simply makes some interventions unavailable, VBID seeks to help payers increase the use of the most appropriate health care interventions, and decrease use of those that do not offer value, on a patient-by-patient basis. ${ }^{14}$ In order to successfully implement VBID, one first needs to understand the value of each product or service. VBP underpins VBID.

In 2013, the UK explored a formal transition to the use of value-based assessment. Although this effort was ultimately abandoned, it was successful in raising awareness and interest in the concept of value-based pricing in medicines globally. ${ }^{15}$ This is mainly driven by the fact that UK drug prices have historically been referenced by many countries. Additionally, National Institute for Health and Care Excellence (NICE) guidelines and recommendations are influential in the decision-making process in several countries. ${ }^{16}$ The extent of this influence highlights the importance of transparency in the methodology behind these decisions, since each payer may place different weightings on the importance of each characteristic of the product. Conceptually, the ultimate goal of 


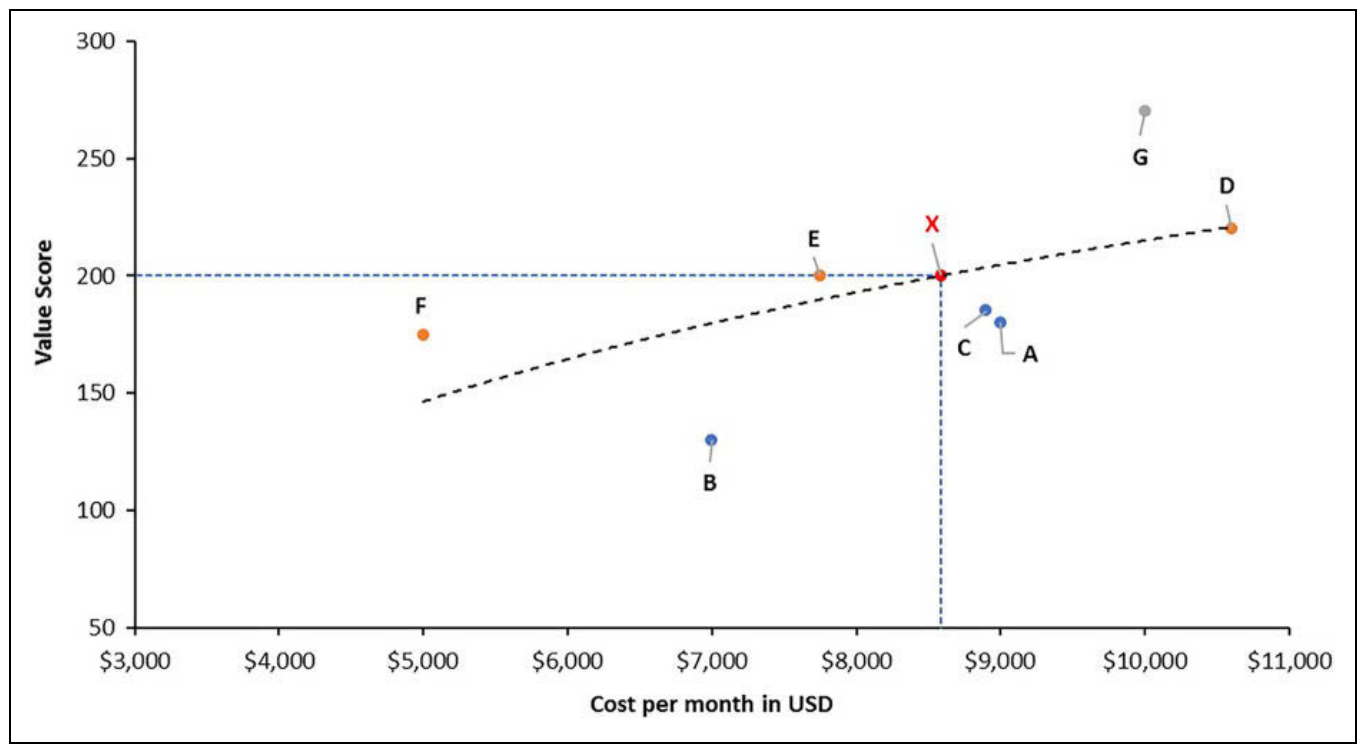

Figure 3. Value vs cost relationship.

value-based pricing is to increase efficiencies by providing the "right" price, increase patient access to treatments that bring true value, and stimulate research and development toward true innovation. The right price is likely to vary by stakeholder.

The robust and transparent EBV framework described above provides a price range but also informs the evidence generation and clinical development processes. The outputs of EBV highlight the clinical and economic evidentiary requirements of the development process, so that trials can be designed around the endpoints that matter to stakeholders. Within the proposed framework, this is achieved through the creation of a payer-specific efficiency curve and clear connections between drug characteristics and price recommendations.

\section{Limitations and Challenges of the EBV Approach}

As with any approach to pricing, there are limitations and challenges associated with this EBV framework. These range from methodological limitations to the challenge of encouraging wide acceptance of EBV.

\section{Methodological Limitations}

- A final value-based price is only as credible as the methodology by which it was derived. While this approach to value-based pricing has been utilized several times in practice by the authors, validating its usefulness, it is still only as good as the attributes on which the EBV framework relies. Product value attributes should be carefully considered in the context of evidence. It is vital to identify the right attributes as these will lead to the most meaningful output. Accordingly, careful examination and interpretation of the existing literature produced by HTA agencies and other stakeholders who have evaluated the product, or similar products, is required.
- EBV relies on the validity of the data collected. An adequate sample size to allow for quantitative analysis and generalizable results is very important but may take some time to fulfill. Study teams must budget enough time and resources to accommodate the correct sample size. This data-gathering effort can be undertaken in a product's development cycle as an iterative process.

- Like many pricing and market research studies, EBV provides estimates that contain uncertainty, and are only as representative and robust as the sample size. Larger sample sizes lead to more reliable results. Price outputs should be interpreted accordingly and standard errors should be considered with any model.

- The Market Adjustment equation variable only captures limited attributes. There are vast numbers of influences in the pharmaceutical market today. The EBV framework only captures those that are measurable and applicable to the specific therapeutic area and product being studied. The data behind the Market Adjustment attributes are primarily based on secondary research and informed by stakeholder interviews. These attributes are not tested in the main conjoint analysis along with the Strength of Differentiation attributes simply because these vary extensively by marketplace and are not, in the authors' experience, major drivers of price nor of great importance to payers.

- With respect to market research limitations, a conjoint exercise is typically conducted to help elucidate the importance of each individual attribute. As with any market research, payers' stated preferences are used to derive pricing premiums. The attribute levels are predefined and then tested with stakeholders, so the EBV model only allows flexibility to test asset profiles within those attribute levels. However, this limitation can easily be addressed by expanding the range of attribute levels 
and increasing respondent sample size. These are important decisions to consider when designing the analysis that will inform the EBV model.

\section{Conclusion}

Macroeconomic pressures and austerity measures are affecting the global marketplace for pharmaceuticals, with health care costs coming under scrutiny like never before. This is reflected in increasing use of HTAs in coverage and reimbursement decisions globally. In more than a dozen countries across Europe as well as in Canada, Australia, and even in emerging markets, national organizations exist to advise on or determine public reimbursement of approved therapies based on formal value appraisals. Biopharma products and medical devices increasingly compete with a broad range of alternative investment opportunities for health care dollars. Ensuring that product price is commensurate with the value it creates through a transparent process to develop pricing will allow for greater access to products and more collaborative discussions with payers.

In the US, as we inch closer toward a consistent implementation of some type of value determination, it is possible that we will eventually converge on a single methodology or some set of methodologies based on a common set of core values. The method we present herein has been utilized in practice on several occasions across a variety of therapeutic areas. It relies on well-documented and clearly implementable methodologies. It can be modified to accommodate products ranging from oncologic therapies designed for end-of-life care, to those to be used for chronic disease management to antibiotics and other acute therapies. We believe that the method described here could be a useful approach for manufacturers and payers to consider as we migrate toward universal implementation of evidence-based medicine in value-based health care systems.

\section{Authors' Note}

This article does not contain any studies with human or animal subjects performed by any of the authors.

\section{Acknowledgments}

The authors thank Jill Dawson, PhD, for medical writing assistance. The authors are all employees of IQVIA (formerly QuintilesIMS).

\section{Declaration of Conflicting Interests}

No potential conflicts were declared.

\section{Funding}

No financial support of the research, authorship, and/or publication of this article was declared.

\section{ORCID iD}

John Doyle, Dr PH, MPH (D) https://orcid.org/0000-0002-4696-2702

\section{References}

1. Knox R. New cancer treatments top $\$ 500,000$ and raise daunting questions about how to pay. http://WBUR.ORG. http:// www.wbur.org/commonhealth/2018/01/11/cancer-drug-costs. Published January 11, 2018. Accessed March 23, 2018.

2. Kirzinger A. Kaiser Health Tracking Poll—late April 2017: the future of the ACA and health care \& the budget. Henry J. Kaiser Family Foundation. https://www.kff.org/report-section/kaiserhealth-tracking-poll-late-april-2017-the-future-of-the-aca-andhealth-care-the-budget-rx-drugs/. Published April 26, 2017. Accessed March 23, 2018.

3. Stinnett AA, Mittleman MA, Weinstein MC, et al. The costeffectiveness of dietary and pharmacologic therapy for cholesterol reduction in adults. In: Gold MR, Siegel JE, Russell LB, Weinstein MC, eds. Cost-Effectiveness in Health and Medicine. New York, NY: Oxford University Press; 1996.

4. Nagle T, Hogan JE, Zale J. The Strategy and Tactics of Pricing: A Guide to Growing More Profitably. 5th ed. Upper Saddle River, NJ: Prentice Hall; 2011. https://books.google.com/books/about/ The_Strategy_and_Tactics_of_Pricing.html?id=20zXQQAA CAAJ. Accessed July 26, 2017.

5. Usman Iqbal SU, Salimi T, Dunlop J, Paramore LC. The early engagement model in product development: linking "proof of concept" to "proof of medical value." Therapeutic Innovation \& Regulatory Science. 2016;50(5):592-601.

6. Elhewaihi E. AMNOG barriers and prospects. ISPOR Connections, 2012. http://www.ispor.org/news/articles/june12/amnogbarriers-and-prospects.asp. Accessed July 26, 2017.

7. ISPOR Global Health Systems Road Map: France-pharmaceuticals. http://www.ispor.org/htaroadmaps/france.asp. Accessed July 26, 2017.

8. Takayama A, Narukawa M. Comparison of new drug accessibility and price between Japan and major European Countries. Therapeutic Innovation \& Regulatory Science. 2017;51(5): 604-611.

9. Presentations at the HTAi Rio de Janeiro Conference 2011, HTA for Health System Sustainability. http:/www.htai.org/meetings/ annual-meetings/past-annual-meetings/htai-2011-rio-de-janeir o.html. Accessed July 26, 2017.

10. Is value truly in the eye of the beholder? Analyzing the heterogeneity of outputs from ASCO, NCCN AND DrugAbacus oncology value frameworks and exploring implications for cancer drug development. Paper presented at: Workshop at ISPOR International Meeting, May 24, 2016.

11. Johnson FR. Patient preferences in regulatory benefit-risk assessments: a US perspective. Value Health. 2016;19(6): 741-745.

12. Postmus D, Mavris M, Hillege HL, et al. Incorporating patient preferences into drug development and regulatory decision making: results from a quantitative pilot study with cancer patients, carers, and regulators. Clin Pharmacol Ther. 2016;99(5): 548-554.

13. European Medicines Agency. Outcome report on pilot to involve patients in benefit/risk discussions at CHMP meetings. http:// 
www.ema.europa.eu/docs/en_GB/document_library/Report/ 2017/05/WC500227335.pdf. Published March 3, 2017. Accessed March 23, 2018.

14. Chernew M, Rosen AB, Fendrick AM. Value-based insurance design. Health Aff. 2007:26(2):w195-w203.
15. Hughes DA. Value-based pricing: incentive for innovation or zero net benefit? Pharmacoeconomics. 2011;29:731-735.

16. Office of Fair Trading. The PPRS: an OFT market study. London: OFT; 2007. https://www.ncbi.nlm.nih.gov/pmc/articles/ PMC1861417/. Accessed November 22, 2016. 